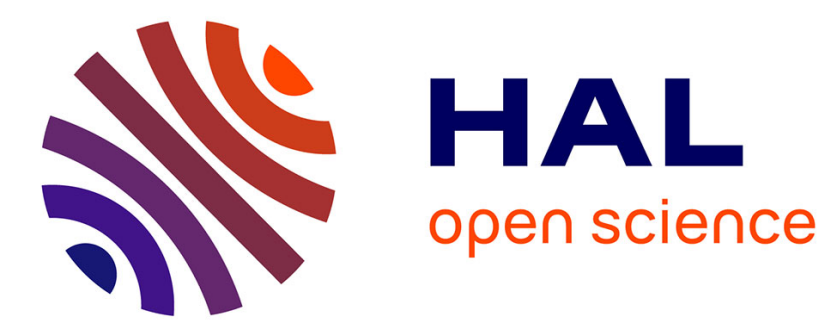

\title{
Traitement des vascularites à ANCA : certitudes et controverses
}

Benjamin Terrier

\section{To cite this version:}

Benjamin Terrier. Traitement des vascularites à ANCA : certitudes et controverses. Néphrologie \& Thérapeutique, 2019, 15, pp.S7 - S12. 10.1016/j.nephro.2019.02.008 . hal-03485004

\section{HAL Id: hal-03485004 https://hal.science/hal-03485004}

Submitted on 20 Dec 2021

HAL is a multi-disciplinary open access archive for the deposit and dissemination of scientific research documents, whether they are published or not. The documents may come from teaching and research institutions in France or abroad, or from public or private research centers.
L'archive ouverte pluridisciplinaire HAL, est destinée au dépôt et à la diffusion de documents scientifiques de niveau recherche, publiés ou non, émanant des établissements d'enseignement et de recherche français ou étrangers, des laboratoires publics ou privés.

\section{다)(1) $(5$}

Distributed under a Creative Commons Attribution - NonCommercial| 4.0 International 
Dochead : Vascularités

\section{Traitement des vascularites à ANCA : certitudes et controverses}

\section{Treatment of ANCA-associated vasculitides: certainties and controversies}

Benjamin Terrier ${ }^{\mathrm{a} * \mathrm{~b}}$

a Service de médecine interne, Centre de références des maladies auto-immunes et systémiques rares, hôpital Cochin, Assistance Publique-Hôpitaux de Paris (AP-HP), 24 rue du Faubourg-Saint-Jacques, 75014 Paris, France

${ }^{\text {b }}$ Faculté de médecine, université Paris Descartes, 24 rue du Faubourg-Saint-Jacques, 75014 Paris, France

*Correspondance : Pr Benjamin Terrier ; tél. : 0158411461 ; fax : 01584114 50 ; e-mail : benjamin.terrier@aphp.fr

\section{Liens d'intérêt}

Roche, Chugaï, Vifor, LFB, Grifols (boards) ; Roche, Chugaï, LFB, Grifols, GSK (consultations) ; Roche, LFB, Grifols, Octapharma, GSK, Janssen (frais de déplacement/congrès) 


\section{Introduction}

Le terme de vascularites systémiques désigne un groupe d'affections caractérisées par une atteinte inflammatoire des vaisseaux sanguins artériels, capillaires et veineux conduisant à une altération de la paroi vasculaire et affectant aussi bien l'endothélium que la média ou l'adventice. En 1990, l'American College of Rheumatology (ACR) a établi une classification des principales vascularites systémiques fondée sur des critères cliniques, biologiques et histologiques. En 1994, la nomenclature de Chapel Hill s'est imposée comme le système de classification de référence et les vascularites ont été alors classées en fonction de la taille des vaisseaux atteints (1). La nomenclature de Chapel Hill a été révisée en 2012, permettant ainsi d'intégrer de nouvelles vascularites systémiques et des outils diagnostiques devenus indispensables en pratique, à savoir la recherche des anticorps anticytoplasme des polynucléaires neutrophiles (antineutrophil cytoplasm antibodies, ANCA) (2). On distingue ainsi : les vascularites des vaisseaux de gros calibre (aorte et ses branches de division), incluant l'artérite de Takayasu et l'artérite à cellules géantes; les vascularites des artères de moyen calibre (principales artères viscérales et leurs branches de division), incluant la périartérite noueuse et la maladie de Kawasaki; les vascularite des vaisseaux de petit calibre (artérioles, capillaires et veinules), comportant les vascularites associées aux ANCA et les vascularites par dépôts de complexes immuns, ces dernières regroupant les vascularites cryoglobulinémiques, les vascularites à immunoglobulines de type A (purpura rhumatoïde), les vascularites urticariennes hypocomplémentémiques (vascularite avec anticorps anti-C1q) et la maladie des anticorps antimembrane basale glomérulaire (anciennement syndrome de Goodpasture). Les vascularites associées aux ANCA regroupent quant à elle la granulomatose avec polyangéite, anciennement maladie de Wegener, la polyangéite microscopique, et la granulomatose éosinophilique avec polyangéite, anciennement syndrome de Churg-Strauss. Le traitement de la granulomatose éosinophilique avec polyangéite ne sera pas abordée ici compte-tenu de la rareté de l'atteinte rénale. Par ailleurs, la granulomatose avec polyangéite et la polyangéite microscopique sont aujourd'hui analysées ensemble dans la plupart des essais thérapeutiques, alors que la granulomatose éosinophilique avec polyangéite fait maintenant l'objet d'études spécifiques.

\section{Caractéristiques des vascularites associées aux ANCA}

\subsection{Granulomatose avec polyangéite (Wegener)}

Il s'agit d'une vascularite associée à des lésions siégeant préférentiellement dans les voies aériennes supérieures, les poumons et les reins. La triade histologique classique de la granulomatose avec polyangéite associe des granulomes, une vascularite nécrosante et/ou granulomateuse des artères et des veines de petits calibres, et une glomérulonéphrite nécrosante segmentaire et focale, avec prolifération extracapillaire sans dépôt d'immunoglobuline ou de complément. La granulomatose avec polyangéite associe ainsi des lésions de vascularite et des lésions granulomateuses, avec une importance variable selon les patients, expliquant la possibilité de voir des présentations différentes selon les patients (3). 
Les lésions de la sphère ORL, particulièrement fréquentes (environ $80 \%$ des cas), se traduisent principalement par une rhinite croûteuse, des épistaxis, une sinusite et/ou une otite séreuse. L'atteinte pulmonaire est également très fréquente ( $70 \%$ à $100 \%$ selon les séries). Cependant, les signes fonctionnels respiratoires sont totalement aspécifiques, allant de l'absence de symptômes à de la toux, des douleurs thoraciques, une hémoptysie, voire une détresse respiratoire. L'atteinte pulmonaire se manifeste par des nodules, unique ou multiples et volontiers excavés, des plages de condensations ou une hémorragie intra-alvéolaire. Les nodules ne sont pas spécifiques et la biopsie est souvent nécessaire pour éliminer un diagnostic différentiel, en particulier un cancer bronchopulmonaire ou une tuberculose.

L’atteinte rénale, caractérisée par une glomérulonéphrite nécrosante extracapillaire pauciimmune se manifeste par un tableau de glomérulonéphrite rapidement progressive associant une protéinurie le plus souvent supérieure ou égale à $1 \mathrm{~g}$ par 24 heures, une hématurie et une dégradation rapide de la fonction rénale. Elle est fréquente, décrite entre 38\% et $100 \%$ selon les séries. Cette atteinte rénale est souvent silencieuse cliniquement au moment du diagnostic ou dans les premières semaines d'évolution de la maladie, justifiant une surveillance régulière de la fonction rénale et du sédiment urinaire dès que le diagnostic est suspecté. Une hématurie microscopique et/ou une protéinurie sont en effet habituellement présentes avant la dégradation de la fonction rénale. La biopsie rénale, lorsqu'elle est pratiquée, peut montrer la coexistence de lésions de glomérulonéphrite aiguë et de cicatrices glomérulaires, témoignant de poussées antérieures, voire des lésions granulomateuses. La présence et l'importance des lésions cicatricielles et sclérosantes sont de moins bon pronostic quant aux capacités de récupération de la fonction rénale sous traitement. La biopsie rénale est ainsi un élément important pour le diagnostic mais aussi pour le pronostic, en évaluant la quantité de ces lésions fibreuses cicatricielles.

La granulomatose avec polyangéite peut se révéler par des signes généraux non spécifiques : la fièvre et/ou altération de l'état général sont présentes chez la plupart des patients, parfois plusieurs semaines ou mois avant que ne s'installe une forme plus bruyante de la maladie. Des myalgies, des arthralgies et plus rarement des arthrites sont souvent observées au moment du diagnostic.

De façon schématique, les granulomatoses avec polyangéite dites « localisées », le plus souvent à la sphère ORL, sont distinguées des granulomatoses avec polyangéite dites « systémiques ». Les formes localisées sont habituellement liées à des manifestations qualifiées de " granulomateuse », menaçant moins le pronostic vital mais plutôt le pronostic fonctionnel, et rechutant plus fréquemment ou pour lesquelles l'obtention d'une rémission complète peut être difficile. Les formes systémiques sont plutôt liées à manifestations dites « vascularitiques » avec des atteintes sévères d'organe, comme une atteinte rénale au premier plan, une hémorragie intra-alvéolaire ou une mononeuropathie multiple. Ces formes systémiques sont initialement plus sévères mais semblent moins rechuter (4). La 
coexistence de manifestations dites « granulomateuses » et « vascularitiques » est fréquente chez un même patient, mais encore une fois, cette caractérisation reste assez schématique et un peu trop simpliste.

Des anticorps anticytoplasme des polynucléaires neutrophiles sont détectés chez $90 \%$ des malades. Ils sont du type C-ANCA et ont une spécificité antiprotéinase 3 dans 75 à $80 \%$ des cas, tandis que dans 10 à $15 \%$ des cas, des anticorps antimyéloperoxidase sont mis en évidence. Enfin, les formes sans ANCA, dites «ANCA négatives » sont possibles dans environ 5 à $10 \%$ des cas.

\subsection{Polyangéite microscopique}

C'est une vascularite des vaisseaux de petit calibre, décrite sans granulome extravasculaire. La polyangéite microscopique a longtemps été confondue avec la périartérite noueuse, alors que les deux maladies sont distinctes, liées à des mécanismes pathogéniques différents.

La polyangéite microscopique peut se révéler par des signes généraux : une fièvre et/ou une altération de l'état général sont présentes chez la plupart des patients, parfois plusieurs semaines ou mois avant que ne s'installe une forme plus bruyante de la maladie. Des myalgies, des arthralgies et plus rarement des arthrites sont observées dans plus de 50\% des cas au moment du diagnostic (5).

L’atteinte rénale, caractérisée par une glomérulonéphrite nécrosante extracapillaire pauciimmune, est l'atteinte décrite le plus souvent, chez environ 75\% des patients (elle est même constante dans certaines séries de patients qui émanent de milieux néphrologiques), et se manifeste par un tableau de glomérulonéphrite rapidement progressive, sans spécificité par rapport à celui observé au cours de la granulomatose avec polyangéite. La biopsie rénale, lorsqu'elle est pratiquée, peut montrer comme au cours de la granulomatose avec polyangéite la coexistence de lésions de glomérulonéphrite aiguë et de cicatrices glomérulaires, témoignant de poussées antérieures. Cependant, les lésions fibreuses, notamment de fibrose interstitielle, semblent spécifiques des malades avec des anticorps antimyéloperoxidase au sein des vascularites associées aux ANCA. La présence et l'importance de ces dernières lésions cicatricielles et sclérosantes sont de moins bon pronostic quant aux capacités de récupération de la fonction rénale sous traitement (6).

L'atteinte pulmonaire est caractérisée par une hémorragie intra-alvéolaire chez environ un tiers des patients atteints de polyangéite microscopique. Associée à la glomérulonéphrite extracapillaire, elle définit le syndrome pneumorénal. Les hémoptysies peuvent être modérées ou massives et responsables de détresse respiratoire, d'anémie, de choc hémorragique. Une fibrose pulmonaire peut être parfois observée aux vascularites ayant des anticorps antimyéloperoxidase. Le lien entre cette fibrose pulmonaire et la vascularite, ou plutôt les ANCA, n'est pas parfaitement défini, mais cette association ne semble pas fortuite. 
Des anticorps anticytoplasme des polynucléaires neutrophiles, habituellement de fluorescence périnucléaire (P-ANCA), sont détectés chez 60 à 75\% des patients, de spécificité antimyéloperoxidase dans la majorité des cas.

\section{3. Évolution et pronostic des vascularites associées aux ANCA}

\subsection{Granulomatose avec polyangéite}

La granulomatose avec polyangéite est une vascularite grave dont l'évolution sans traitement conduit constamment au décès. Sous traitement, le pronostic vital s'est considérablement amélioré, avec actuellement une survie à 10 ans supérieure à 75\%. L'évolution de la granulomatose avec polyangéite est marquée par des rechutes très fréquentes, la moitié des malades rechutant dans les 5 ans suivant la première poussée de la maladie (4). La fréquence de ces rechutes implique un poids du traitement et des complications iatrogènes. Les infections et les poussées sévères de la maladie sont les causes principales de décès dans la première année de traitement, tandis que les complications cardiovasculaires sont la cause principale de décès à long terme (7). Les facteurs de mauvais pronostic au moment du diagnostic initial de la granulomatose avec polyangéite sont une créatininémie supérieure à $150 \mu \mathrm{mol} / \mathrm{L}$, une cardiomyopathie spécifique, un âge de plus de 65 ans, une atteinte digestive grave et l'absence d'atteinte ORL (5). Les malades ont fréquemment des séquelles ORL avec la persistance de croûtes nasales et d'épistaxis alors que la vascularite est contrôlée par ailleurs, on parle alors de maladie «grumbling ». La surdité est une séquelle invalidante de la maladie. L'évolution vers l'insuffisance rénale terminale est la complication redoutée des atteintes rénales sévères ou répétées.

\subsection{Polyangéite microscopique}

L'évolution de la polyangéite microscopique peut être émaillée de rechutes, avec environ un tiers des patients rechutant après l'obtention d'une rémission complète. Il n'est cependant pas encore possible d'identifier clairement le sous-groupe de patients rechuteurs. La mortalité globale des patients atteints de polyangéite microscopique est de l'ordre de $30 \%$ à 5 ans. Cependant, la plupart des décès surviennent chez les patients atteints des formes les plus sévères, ayant un ou plusieurs facteurs de mauvais pronostic selon le Five Factor Score (créatininémie supérieure à $150 \mu \mathrm{mol} / \mathrm{L}$, atteinte myocardique, atteinte digestive ou âge de plus de 65 ans). Comme au cours de la granulomatose avec polyangéite, le décès peut être secondaire à une poussée de la maladie mais aussi à la toxicité des traitements (corticoïdes et/ou immunosuppresseurs), en particulier les infections graves ou les complications cardiovasculaires.

\section{Traitement des vascularites associées aux ANCA}

Le traitement des vascularites associées aux ANCA dépend du type de vascularite, de la sévérité de la maladie, et bien entendu du terrain et des comorbidités potentielles du patient pris en charge. 
L'association des glucocorticoïdes avec un immunosuppresseur ou un immunomodulateur est toujours le traitement de référence de la granulomatose avec polyangéite. Cette association est aussi nécessaire dans les formes graves de polyangéite microscopique. Dans les formes peu sévères de cette dernière pathologie, sans facteur de mauvais pronostic, une corticothérapie seule est habituellement prescrite, mais une étude prospective randomisée contrôlée devrait prochainement débutée en France, conduite par le Groupe français d'étude des vascularites (GFEV), pour répondre à la question de l'intérêt du rituximab dans ces formes «non sévères » de polyangéite microscopique.

Le traitement combinant un glucocorticoïde et le cyclophosphamide était considéré comme le « gold standard » jusqu'en 2010, année où un le rituximab a démontré sa non-infériorité.

\subsection{Traitement d'induction}

\subsubsection{Glucocorticö̈des}

La dose journalière de prednisone est initialement de $1 \mathrm{mg} / \mathrm{kg} / \mathrm{jour}$, sans dépasser habituellement 60 à $80 \mathrm{mg} / \mathrm{j}$ our, et peut être précédée, selon la gravité de la maladie, d'un ou plusieurs bolus de méthylprednisolone (7,5 à $15 \mathrm{mg} / \mathrm{kg}$ par jour). Après un traitement de 2 à 4 semaines, la posologie du médicament doit être réduite et la corticothérapie maintenue pendant 12 à 18 mois.

Bien qu'ils ne soient pas encore publiés, les résultats de l'essai PEXIVAS ont montré qu'un schéma de corticoïdes réduit (avec notamment une dose de prednisone de $10 \mathrm{mg} / \mathrm{j}$ à 3 mois et $5 \mathrm{mg} / \mathrm{j}$ à 6 mois) était associé à la survenue d'un décès et/ou d'une insuffisance rénale chronique terminale chez $26 \%$ dans le groupe « corticothérapie standard » comparativement à $28 \%$ dans le groupe « corticothérapie réduite » (hypothèse de non-infériorité vérifiée). En revanche, il y avait moins d'infections sévères au cours de la première année dans le groupe avec dose réduite de corticoïdes (rapport de taux d'incidence de 0,70, intervalle de confiance à 95\% [IC 95\%] de 0,52 à 0,94; $p=0,02$ ).

Des méta-analyses ont suggéré néanmoins, afin de réduire la fréquence des rechutes, qu'il serait souhaitable de prolonger l'administration de prednisone au-delà de cette période, mais à faible dose (5 $\mathrm{mg} / \mathrm{j}$ ) (8). Deux études randomisées contrôlées, une américaine en cours et une française qui devrait bientôt débuter, tenteront de préciser la durée optimale de la corticothérapie.

Une étude internationale évaluant le CCX168 comparativement à la corticothérapie, donc incluant un bras sans glucocorticoïdes, est en cours et permettra de voir si des schémas sans corticoïdes sont possibles.

\subsubsection{Cyclophosphamide}

Des bolus de cyclophosphamide sont proposés en traitement initial, associés à la corticothérapie, pour induire la rémission des vascularites. Le cyclophosphamide est prescrit au cours de la granulomatose 
avec polyangéite et la polyangéite microscopique initialement toutes les 2 semaines pendant un mois, à la dose de $0,6 \mathrm{~g}$ par $\mathrm{m}^{2}$, puis de $0,7 \mathrm{~g}$ par $\mathrm{m}^{2}$ toutes les 3 semaines.

Chez les patients avec fonction rénale normale et âgés de moins de 65 ans, le cyclophosphamide est prescrit par voie intraveineuse à la dose de $0,6 \mathrm{~g} / \mathrm{m}^{2}$ à $\mathrm{j} 1, \mathrm{j} 15$ et $\mathrm{j} 29$, puis $0,7 \mathrm{~g} / \mathrm{m}^{2}$ tous les 21 jours (pour totaliser six à neuf bolus en fonction de la réponse). En revanche, chez les patients avec un débit de filtration glomérulaire inférieur à $30 \mathrm{ml} / \mathrm{min}$ et âgés de moins de 65 ans, le cyclophosphamide est prescrit à la dose de $0,5 \mathrm{~g} / \mathrm{m}^{2}$ tant que le débit de filtration glomérulaire est inférieur $30 \mathrm{ml} / \mathrm{min}$. Enfin, chez les patients âgés de plus de 65 ans, le cyclophosphamide est prescrit à la dose de $0,5 \mathrm{~g}$ à dose fixe à j1, j15 et j29, puis tous les 21 jours selon les données de l'essai CORTAGE (9).

\subsubsection{Rituximab}

L'étude RAVE a marqué un réel tournant dans le traitement d'induction de la granulomatose avec polyangéite et de la polyangéite microscopique en proposant le rituximab comme une alternative efficace au cyclophosphamide (10,11). Au cours des vascularites associées aux ANCA, le schéma validé repose sur des perfusions de rituximab de $375 \mathrm{mg} / \mathrm{m}^{2} /$ semaine pendant 4 semaines consécutives, avec une non-infériorité comparativement au cyclophosphamide en termes d'efficacité et un profil de tolérance comparable.

Dans plusieurs études rétrospectives, un schéma d'administration de $1 \mathrm{~g}$ à j1 et j15 a été utilisé, sans événement indésirable particulier, ni infériorité évidente. Une seule étude rétrospective, de faible effectif, a comparé l'efficacité du schéma administrant $375 \mathrm{mg} / \mathrm{m}^{2} / \mathrm{semaine}$ pendant 3 semaines consécutives au schéma administrant $1 \mathrm{~g}$ à $\mathrm{j} 1$ et j15, sans mettre en évidence de différence d'efficacité (12). Cependant, les patients inclus dans cette étude étaient essentiellement porteurs d'ANCA antiprotéinase 3, ne permettant pas d'avoir de donnée chez les patients avec ANCA antimyéloperoxidase. Le schéma administrant $375 \mathrm{mg} / \mathrm{m}^{2} /$ semaine pendant 3 semaines consécutives (quatre perfusions à une semaine d'intervalle) reste cependant le seul schéma validé et donc recommandé.

\subsubsection{Choix entre le cyclophosphamide et le rituximab}

La question du choix préférentiel entre le cyclophosphamide et le rituximab est une question courante en pratique.

Dans l'étude de référence, le rituximab n'a pas été comparé au cyclophosphamide chez les patients présentant une hémorragie alvéolaire requérant une ventilation mécanique, ou chez les patients ayant une insuffisance rénale rapidement progressive avec une créatininémie supérieure à 350 $\mu \mathrm{mol} / \mathrm{L}$ (11). Ainsi, il est difficile aujourd'hui de recommander le rituximab dans ces formes non évaluées comparativement au cyclophosphamide. 
Une autre étude prospective, randomisée, a comparé une association de corticoïdes, de cyclophosphamide et de rituximab à l'association de corticoïdes et de cyclophosphamide en traitement d'induction des vascularites rénales sévères (13). Elle ne trouvait pas de supériorité d'un traitement par rapport à l'autre. Les résultats de cette étude suggèrent que dans ces situations sévères, l'association de corticoïdes, de cyclophosphamide et de rituximab pourrait être utilisée dans certaines situations.

En revanche, chez les patients ayant déjà rechuté et/ou qui ont déjà̀ reçu du cyclophosphamide, et chez les patients en âge de procréer, l'utilisation préférentielle du rituximab semble assez justifiée (14).

\subsection{5. Échanges plasmatiques}

Les échanges plasmatiques thérapeutiques, associés aux corticoïdes et aux immunosuppresseurs, ont été prescrits depuis plusieurs décennies pour traiter des vascularites nécrosantes systémiques. Aucune étude rétrospective ou prospective n'a montré d'amélioration de la survie chez ces patients $(13,15,16)$. Seule l'étude comparant bolus de méthylprednisolone et échanges plasmatiques en adjonction aux corticoïdes et au cyclophosphamide, chez les patients ayant une vascularites associées aux ANCA avec une glomérulonéphrite extracapillaire se manifestant par une créatininémie initiale supérieure à $500 \mu \mathrm{mol} / \mathrm{L}$, a montré un bénéfice significatif sur la fonction rénale à court terme (inférieur à 12 mois) (16).

Les résultats négatifs de l'essai international PEXIVAS, non encore publiés, évaluant l'intérêt des échanges plasmatiques thérapeutiques au cours des vascularites à ANCA avec une insuffisance rénale définie par un débit de filtration glomérulaire estimé inférieur à $50 \mathrm{ml} / \mathrm{min} / 1,73 \mathrm{~m}^{2}$ et/ou une hémorragie intra-alvéolaire, ont remis en question leur place dans la prise en charge des patients. Le conseil scientifique du GFEV (dont les membres sont Vincent Cottin, Stanislas Faguer, Loïc Guillevin, Noémie Jourde-Chiche, Alexandre Karras, Luc Mouthon, Antoine Néel, Xavier Puéchal, Grégory Pugnet, Maxime Samson, Camille Taillé, Benjamin Terrier), s’est ainsi positionné sur la place des échanges plasmatiques thérapeutiques, dans l'attente de la publication définitive, livrant le texte ci-dessous.

L'essai international PEXIVAS ayant inclus 704 patients a voulu répondre à la question de l'intérêt des échanges plasmatiques thérapeutiques dans une population de patients avec vascularites associées aux ANCA atteints d'une insuffisance rénale définie par un débit de filtration glomérulaire estimé inférieur à $50 \mathrm{ml} / \mathrm{min} / 1,73 \mathrm{~m}^{2}$ et/ou une hémorragie intra-alvéolaire.

Les patients étaient répartis aléatoirement soit dans le bras avec échange plasmatique (sept échanges de $60 \mathrm{ml} / \mathrm{kg}$ sur 14 jours) soit dans le bras contrôle sans échange, puis recevaient des bolus de méthylprednisolone (1,5 à $3 \mathrm{~g}$ ), et étaient ensuite répartis aléatoirement une nouvelle fois soit dans un groupe recevant des corticoïdes à dose standard soit dans un groupe recevant des corticoïdes à une 
dose réduite d'environ 60\%. Quatre-vingt-dix-huit pour cent des patients inclus avaient une insuffisance rénale, tandis que 27\% étaient atteints d'hémorragie alvéolaire, dont seulement 8 à $9 \%$ avaient une hémorragie intra-alvéolaire considérée comme sévère. Le principal résultat préliminaire de cet essai concernant l'intérêt des échanges plasmatiques thérapeutiques a montré la survenue d'un décès et/ou d'une insuffisance rénale chronique terminale chez $28 \%$ des patients du groupe avec échanges plasmatiques thérapeutiques contre $31 \%$ des patients sans échange (hazard ratio de 0,86 ; IC 95\%:0,65-1,13; $p=0,27)$. Aucune des analyses en sous-groupes, ni l'analyse séparée de la mortalité ou de la survenue d'une insuffisance rénale chronique terminale ne retrouvaient de bénéfice significatif des échanges plasmatiques thérapeutiques.

L'analyse des patients ayant une hémorragie alvéolaire semblait cependant montrer un signal en faveur des échanges plasmatiques thérapeutiques, avec un risque relatif d'atteindre le critère primaire composite de jugement (décès et/ou insuffisance rénale chronique terminale) de 0,95 en l'absence d'hémorragie, 0,65 en présence d'une hémorragie modérée et 0,67 en présence d'une hémorragie sévère (différence non significative). Aussi, chez les patients ayant une hémorragie alvéolaire sévère, dont la plupart avaient également une atteinte rénale, la mortalité semblait moindre dans le groupe avec échanges plasmatiques thérapeutiques (11 patients sur 31 [soit 35\%] sans échange, contre six patients sur 30 [soit 20\%] dans le groupe avec échanges plasmatiques thérapeutiques).

En définitive, les résultats préliminaires de l'essai PEXIVAS montrent l'absence de bénéfice des échanges plasmatiques thérapeutiques sur la mortalité et/ou la survenue d'une insuffisance rénale chronique terminale au cours des vascularites associées aux ANCA.

Parmi les différents éléments à prendre en compte dans l'interprétation des résultats, notamment des sous-groupes il était important de noter que :

- un essai randomisé contrôlé est conçu pour répondre à l'objectif principal de l'étude (qui était ambitieux dans l'essai PEXIVAS, avec une réduction de 35\% du risque relatif du critère principal), et le niveau de preuve des résultats issus des analyses en sous-groupe est toujours inférieur à ceux pour l'objectif principal ;

- contrairement à l'essai MEPEX, l'ensemble des patients a reçu des bolus de méthylprednisolone et le recul moyen par rapport à l'intervention thérapeutique était de plusieurs années, ce qui peut peut-être expliquer certaines différences avec les résultats de l'essai MEPEX ;

- nous n'avons pour l'instant pas d'information concernant le mode de présentation de l'atteinte rénale (aiguë ou progressive) et le type d'atteinte histologique (présence de croissants, formes granulomateuses, séquelles fibreuses à l'admission, degré de nécrose tubulaire aiguë, etc.), variables qui peuvent modifier grandement la réponse attendue aux échanges plasmatiques thérapeutiques ; 
- un biais d'inclusion possible ayant impacté le résultat de cet essai ne peut pas être exclu, les investigateurs ayant pu être hésitants à sélectionner un patient dont le pronostic vital était mis en jeu, compte tenu des résultats antérieurs de l'essai MEPEX ;

- $\quad$ il n’y a pas de données dans certaines formes cliniques sévères (vascularites cérébrales, myocardites, etc.).

Ainsi, le conseil scientifique du GFEV pense que même si l'utilisation des échanges plasmatiques thérapeutiques doit désormais être réduite, leur intérêt chez certains patients ne peut pas être exclu, après discussion au cas par cas, en particulier :

- les patients ayant une hémorragie alvéolaire sévère ;

- les patients ayant une aggravation persistante de leur insuffisance rénale malgré le traitement conventionnel par corticoïdes associés au cyclophosphamide ou au rituximab ;

- les patients atteints d'une glomérulonéphrite rapidement progressive et/ou d'une hémorragie alvéolaire sans diagnostic de certitude, au moins jusqu'au résultat de la recherche d'anticorps antimembrane basale glomérulaire et/ou du diagnostic de certitude (avec un éventuel arrêt une fois le diagnostic de vascularites associées aux ANCA posé).

Bien entendu, l'article définitif n'étant pas encore publié, il n'est pas exclu que cet avis soit amené à évoluer dans le futur.

\subsection{Traitement d'entretien}

Au cours de la granulomatose avec polyangéite et de la polyangéite microscopique sévère, les résultats d'un essai prospectif coordonné par le GFEV et comparant le rituximab à l'azathioprine en traitement d'entretien de la rémission ont démontré la supériorité du rituximab pour prévenir la survenue de rechutes, selon un schéma de rituximab à la dose de $500 \mathrm{mg}$ fixe à j0, j15, 6 mois, 12 mois et 18 mois pour prévenir des rechutes majeures (17). Une extension d'autorisation de mise sur le marché pour l'utilisation du rituximab en traitement d'entretien a été obtenue auprès de la Food and Drug Administration américaine et de l'agence européenne des médicaments (European Medicines Agency, EMA) en 2018.

Le suivi à long terme de cet essai a néanmoins montré la survenue de rechutes tardives, essentiellement chez les patients avec ANCA antiprotéinase 3, démontrant l'intérêt d'optimiser ce schéma thérapeutique (18). Les résultats de l'essai comparant le rituximab à date fixe décrit ci-dessus et le rituximab « à la demande » en fonction du titre des ANCA et/ou de la présence de CD19 ont montré que le taux de rechute n'était pas différent entre les deux stratégies (19). Un essai thérapeutique en cours compare, après l'administration de rituximab d'entretien pendant 18 mois, soit quatre perfusions supplémentaires de rituximab à 6 mois d'intervalle, soit des perfusions de placebo. Il 
permettra de savoir si un traitement plus prolongé est justifié en première intention, en particulier chez les patients ayant des ANCA antiprotéinase 3.

L'azathioprine et le méthotrexate n'ont ainsi de place en traitement d'entretien que chez les patients présentant une intolérance et/ou une contre-indication au rituximab, ou en cas d'échec du rituximab en traitement d'entretien (20).

Enfin, la question de la poursuite ou non du traitement d'entretien chez les patients ayant atteint l'insuffisance rénale chronique terminale est un point de controverse entre néphrologues et internistes notamment, la poursuite du traitement pouvant exposer ces patients à risque à des complications infectieuses, mais l'arrêt pouvant les exposer à l'inverse à des rechutes potentiellement graves.

L'essai prospectif randomisé MASTER-ANCA, a ainsi pour objectif d'évaluer la faisabilité, le risque et les avantages liés à l'abandon du traitement d'entretien immunosuppresseur dans cette population de patients. Les patients atteints d'insuffisance rénale terminale liée à la vascularite seront répartis aléatoirement entre deux stratégies : arrêt (ou non initiation) du traitement d'entretien (groupe expérimental) ou maintien (ou initiation) du traitement immunosuppresseur (groupe témoin). Il s'agit d'une étude de supériorité de l'arrêt du traitement par rapport au maintien.

\section{Conclusion}

Le traitement des vascularites associées aux ANCA a beaucoup évolué au cours de ces dernières années, en particulier en diminuant fortement le risque de rechutes avec l'utilisation du rituximab. Outre les séquelles liées à la maladie initiale et aux rechutes, le pronostic est également grévé par les complications des traitements, au premier rang desquels la corticothérapie. Les essais thérapeutiques futurs auront ainsi pour but de limiter l'utilisation des corticoïdes tout en maintenant un bon contrôle de la maladie.

\section{Références}

1. Jennette JC, Falk RJ, Andrassy K, Bacon PA, Churg J, Gross WL, et al. Nomenclature of systemic vasculitides. Proposal of an international consensus conference. Arthritis Rheum 1994;37(2):187-92.

2. Jennette JC, Falk RJ, Bacon PA, Basu N, Cid MC, Ferrario F, et al. 2012 revised International Chapel Hill Consensus Conference Nomenclature of Vasculitides. Arthritis Rheum 2013;65(1):1-11.

3. Puéchal X, Pagnoux C, Perrodeau É, Hamidou M, Boffa J-J, Kyndt X, et al. Long-term outcomes among participants in the WEGENT trial of remission-maintenance therapy for 
granulomatosis with polyangiitis (Wegener's) or microscopic polyangiitis. Arthritis Rheumatol 2016;68(3):690-701.

4. Holle JU, Gross WL, Latza U, Nölle B, Ambrosch P, Heller M, et al. Improved outcome in 445 patients with Wegener's granulomatosis in a German vasculitis center over four decades. Arthritis Rheum 2011;63(1):257-66.

5. Guillevin L, Pagnoux C, Seror R, Mahr A, Mouthon L, Le Toumelin P, et al. The Five-Factor Score revisited: assessment of prognoses of systemic necrotizing vasculitides based on the French Vasculitis Study Group (FVSG) cohort. Medicine 2011;90(1):19-27.

6. Kapitsinou PP, Ioannidis JPA, Boletis JN, Sotsiou F, Nakopoulou L, Daphnis E, et al. Clinicopathologic predictors of death and ESRD in patients with pauci-immune necrotizing glomerulonephritis. Am J Kidney Dis 2003;41(1):29-37.

7. Flossmann O, Berden A, de Groot K, Hagen C, Harper L, Heijl C, et al. Long-term patient survival in ANCA-associated vasculitis. Ann Rheum Dis 2011;70(3):488-94.

8. Walsh M, Merkel PA, Mahr A, Jayne D. Effects of duration of glucocorticoid therapy on relapse rate in antineutrophil cytoplasmic antibody-associated vasculitis: A meta-analysis. Arthritis Care Res 2010;62(8):1166-73.

9. Pagnoux C, Quéméneur T, Ninet J, Diot E, Kyndt X, de Wazières B, et al. Treatment of systemic necrotizing vasculitides in patients aged sixty-five years or older: results of a multicenter, open-label, randomized controlled trial of corticosteroid and cyclophosphamide-based induction therapy. Arthritis Rheumatol 2015;67(4):1117-27.

10. Specks U, Merkel PA, Seo P, Spiera R, Langford CA, Hoffman GS, et al. Efficacy of remission-induction regimens for ANCA-associated vasculitis. N Engl J Med 2013;369(5):417-27.

11. Stone JH, Merkel PA, Spiera R, Seo P, Langford CA, Hoffman GS, et al. Rituximab versus cyclophosphamide for ANCA-associated vasculitis. N Engl J Med 2010;363(3):221-32.

12. Jones RB, Ferraro AJ, Chaudhry AN, Brogan P, Salama AD, Smith KGC, et al. A multicenter survey of rituximab therapy for refractory antineutrophil cytoplasmic antibody-associated vasculitis. Arthritis Rheum 2009;60(7):2156-68. 
13. Rifle G, Chalopin JM, Zech P, Deteix P, Ducret F, Vialtel P, et al. Treatment of idiopathic acute crescentic glomerulonephritis by immunodepression and plasma-exchanges. A prospective randomised study. Proc Eur Dial Transpl Assoc 981;18:493-502.

14. Charles P, Bienvenu B, Bonnotte B, Gobert P, Godmer P, Hachulla É, et al. Rituximab: Recommendations of the French Vasculitis Study Group (FVSG) for induction and maintenance treatments of adult, antineutrophil cytoplasm antibody-associated necrotizing vasculitides. Presse Med 2013;42(10):1317-30.

15. Guillevin L, Fain O, Lhote F, Jarrousse B, Le Thi Huong D, Bussel A, et al. Lack of superiority of steroids plus plasma exchange to steroids alone in the treatment of polyarteritis nodosa and Churg-Strauss syndrome. A prospective, randomized trial in 78 patients. Arthritis Rheum 1992;35(2):208-15.

16. Jayne DRW, Gaskin G, Rasmussen N, Abramowicz D, Ferrario F, Guillevin L, et al. Randomized trial of plasma exchange or high-dosage methylprednisolone as adjunctive therapy for severe renal vasculitis. J Am Soc Nephrol 2007;18(7):2180-8.

17. Guillevin L, Pagnoux C, Karras A, Khouatra C, Aumaître O, Cohen P, et al. Rituximab versus azathioprine for maintenance in ANCA-associated vasculitis. N Engl J Med 2014;371(19):1771-80.

18. Terrier B, Pagnoux C, Perrodeau É, Karras A, Khouatra C, Aumaître O, et al. Long-term efficacy of remission-maintenance regimens for ANCA-associated vasculitides. Ann Rheum Dis 2018;77(8):1150-1156.

19. Charles P, Terrier B, Perrodeau É, Cohen P, Faguer S, Huart A, et al. Comparison of individually tailored versus fixed-schedule rituximab regimen to maintain ANCA-associated vasculitis remission: results of a multicentre, randomised controlled, phase III trial (MAINRITSAN2). Ann Rheum Dis 2018;77(8):1143-1149.

20. Pagnoux C, Mahr A, Hamidou MA, Boffa J-J, Ruivard M, Ducroix J-P, et al. Azathioprine or methotrexate maintenance for ANCA-associated vasculitis. N Engl J Med 2008;359(26):2790-803.

21. Jones RB, Tervaert JWC, Hauser T, Luqmani R, Morgan MD, Peh CA, et al. Rituximab versus cyclophosphamide in ANCA-associated renal vasculitis. N Engl J Med 2010;363(3):211-20. . 